\title{
Assessment of Quality of life Among Children with Cancer, Children Undergoing Hemodialysis and Children with Thalassemia: A Comparative Study
}

Tayseer Afifi ( $\square$ afifi.tayseer@gmail.com )

Islamic University of gaza https://orcid.org/0000-0001-5289-9538

Khamis Elessi

Islamic University of Gaza

Obay Baraka

Islamic University of Gaza

Mohammed Omar

Islamic University of Gaza

Israa Ahmad

Islamic University of Gaza

Hala Al-Attar

Islamic University of Gaza

Ameera Qudaih

Al Azhar University - Gaza

Khalid Alsultan

Islamic University of Gaza

Meral Abdelati

Islamic University of Gaza

Alaa Astal

Islamic University of Gaza

Samar Abu Shammala

Islamic University of Gaza

Mohammed Obaid

Islamic University of Gaza

Mohammed Abu-Rous

Islamic University of Gaza

Mosab Samaan

Islamic University of Gaza

Khalil Albatsh

Islamic University of Gaza

Abdarrahman Almoqaid 
Islamic University of Gaza

\section{Research}

Keywords: quality of life, child, cancer, hemodialysis, thalassemia, Gaza

Posted Date: February 17th, 2020

DOI: https://doi.org/10.21203/rs.2.23686/v1

License: (c) (i) This work is licensed under a Creative Commons Attribution 4.0 International License. Read Full License 


\section{Abstract}

Purpose This study aims to evaluate health-related quality of life among children with different chronic conditions and make a comparison among them. In addition, the study will draw a comparison between children's perspective about their quality of life and parents' perspective. Furthermore, the study will address the associations between quality of life with disease severity, duration and the presence of other co-morbidities.

Methods This was a prospective, analytical, correlational study design in which Pediatric Quality of Life Inventory (PedsQL 4.0 generic core scale) was administered to assess quality of life on 110 children; 50 children with cancer, 30 children with thalassemia and 30 children undergoing hemodialysis. Researchers interviewed children as well as their parents.

Results Results showed that children with Thalassemia had the lowest scores and suffered from poor quality of life compared to the other two groups of children. Interestingly, quality of life level among children undergoing dialysis was lower than children with cancer. However, there were marked discrepancy between child's and parents' answers on scale questions. The level of quality of life, in all children, were statistically significant in relation with family size, income and parents' education.

Conclusion We identified high prevalence of poor level of health-related quality of life among children included in this study. The findings support that the quality of life among children with chronic conditions should receive more attention in our local medical settings.

\section{Introduction}

Children with cancer show a marked reduction in quality of life (QoL) in the acute phase of their disease according to the international literature ${ }^{[1-3]}$. Quality of life is a subjective concept. Some define it as the feeling of socioemotional and physical well-being, including the ability to participate in everyday activities according to age ${ }^{[4]}$. In children with cancer, thalassemia or children undergoing hemodialysis, QoL develops as a personal experience, influenced by its sickness and the institutional environments in which they spend their daily life ${ }^{[4]}$. The sickness stigma and the aggressiveness of the received medical treatments among those children are factors that affect their development, generating alterations at physical and psychological levels ${ }^{[5]}$. Considering this, it is necessary to understand that QoL and health are aspects strongly related, because sickness has as important impact on the individual well-being and its ability to function in physical, emotional and social areas in the daily life ${ }^{[4]}$.

Diagnosis with cancer can have a significant impact on life conditions of any individual. However, this is more prevalent among vulnerable groups such as children. It doesn't only affect child's life, but also the whole family. Furthermore, not only cancer that changes child's life, but also other chronic conditions may have more devastating effect. Children undergoing hemodialysis could be exposed to depressive symptoms almost same as children with cancer do. In addition, children with thalassemia, who cannot 
have a normal daily activity due to the fear of any injury that may cause life-threatening bleeding, are even more susceptible to changes in their quality of life.

Cancer is the second leading cause of death globally and is estimated to account for 9.6 million death in $2018^{[6]}$. Among children in Gaza Strip, $6.6 \%$ of deaths are due to cancer. While renal failure caused $1.3 \%$ of deaths in $2017^{[7]}$. Thalassemia affects approximately 4.4 of every 10,000 live births throughout the world ${ }^{[8]}$. In Palestine, $4 \%$ of the population are known to be thalassemia carriers with new cases continuing to appear despite the availability of prenatal testing ${ }^{[9]}$.

A lot of studies, locally and internationally, investigated QoL among oncology pediatric patients. However, few studies were found to address this topic among children undergoing hemodialysis or children with thalassemia. In an observational, analytical study ${ }^{[10]}$ conducted among Pakistani children with thalassemia using transfusion-dependent QoL (TranQoL) questionnaire, it was found that quality of life was affected not only by clinical conditions, but also life alteration, social relationships and psychological events. Another study [11], compared between children undergoing peritoneal dialysis versus children undergoing hemodialysis, found that QoL was significantly higher among children undergoing peritoneal dialysis. Furthermore, male children undergoing hemodialysis had better quality of life that female children in the same group.

The lack of research investigating the well-being and health-related quality of life among children with cancer, thalassemia and children undergoing hemodialysis encouraged this research. This study aims to (1) assess the quality of life among these children and draw a comparison between study sample groups, (2) to determine the factors that influence children's life conditions, (3) to explore the association between quality of life and disease severity, duration and the presence of other co-morbidities and finally (4) to compare between child's perspective and parents' perspective on level of quality of life among children.

\section{Methods}

\section{Research Design}

The design of this study is descriptive, correlational, analytical and cross-sectional design. As the study aims to determine the quality of life level among study population, this is the most appropriate design.

\section{Setting of the study}

The study took place at two hospitals in Gaza Strip. These hospitals were Elrantessy Specialized Pediatric Hospital in Gaza city, which is the main center for pediatric cancer patients, and European Gaza Hospital.

\section{Study sample}

The study included children between 7-12 years of age and their parents. The sample was as follows: 50 children with cancer, 30 children with thalassemia and 30 children undergoing hemodialysis at three 
hospitals in Gaza Strip. Researchers interviewed children as well as their parents. All interviewed parents were mothers. The sample was chosen according to the inclusion criteria in the next paragraph.

\section{Eligibility criteria}

The following criteria must be met in the child in order to be included in study sample.

- Age 7-12 years.

- The child is diagnosed with cancer or other chronic conditions, namely: renal, or Thalassemia.

- The child doesn't suffer from any documented mental illness.

- The child is able to respond to study questions.

- Child's parents must attend the interview.

\section{Data collection}

Initially, a team composed of 10 medical students was trained on study protocol and interview questions. After getting the permission, face to face interviews with children and their parents were conducted in the above-mentioned hospitals. An informed consent was gained from parents.

\section{Operational definitions}

Children in this study are individuals aged between $7-13$ years as mentioned the eligibility criteria. World Health Organization (WHO) defines Quality of Life as an individual's perception of their position in life, according to age, in the context of the culture and value systems in which they live and in relation to their goals, expectations, standards and concerns ${ }^{[12]}$.

\section{Instruments}

The psychometric tools included in the study are presented below.

1. A semi-structured questionnaire concerning demographic data (age, gender, gestational age, birth weight, disease history, education, etc....).

2. Pediatric Quality of Life Inventory (PedsQL 4.0 generic core scale) was used for this study to assess QOL. The PedsQL scale is interviewer-administrated QOL scoring system. The 23-item multidimensional PedsQL 4.0 Generic Core Scales encompass 4 scales: physical functioning, emotional functioning, social functioning, and school functioning. The participants evaluated how often a specific problem occurred in the past month, A 5-point Likert response scale is utilized across child self-report for ages $7-12$ years $(0=$ never a problem; $1=$ almost never a problem; $2=$ sometimes a problem; 3 = often a problem; 4 = almost always a problem). The Arabic version was validated and showed high reliability with Cronbach's alpha coefficient of $0.86^{[13]}$.

\section{Statistical Analysis}


Data obtained were coded and entered into Statistical Package for Social Sciences (SPSS). Categorical variables were presented as frequency and percentage while continuous variables were presented as mean and standard deviation. Spearman correlation test was used to test for relationship with continuous variables while Independent sample T-test was used to determines whether there is a statistically significant difference between the means in study groups.

\section{Permission and ethical considerations}

An approved permission was gained from Ministry of Health in Gaza Strip to conduct interviews with pediatric patients and their parents. Furthermore, informed consents were also gained from parents and any unclear questions will be simplified for the participant.

\section{Results}

\section{Baseline characteristics of children and their parents}

Study participants were classified into three groups: children with cancer, children with thalassemia and children undergoing hemodialysis. Mean age among children was $9 \pm 2.5$ years and all of them were in the age group 7-13 years. Among all children, 64 (58\%) were males and most of children attend school $(n=101,92 \%)$. More details on sociodemographic data of study sample are provided in table 1.

Type of cancer varied among children. The most prevalent type was leukemia followed by neuroblastoma and then renal tumor.

\section{Quality of life assessment among children}

Children with thalassemia had the lowest level of quality of life $(n=21,70 \%)$, in general, followed by children undergoing hemodialysis $(n=19,63.3 \%)$ and lastly children with cancer $(n=29,58 \%)$. The physical functioning scale was the lowest scores among children with thalassemia. On the other hand, children with cancer and children undergoing hemodialysis showed good scores in the physical functioning scale, but low scores were evident in the emotional functioning scale. All children had good levels in both social and school functioning scales. Figure 1 illustrates children's scores' mean values in the 4 scales.

It is observed that all children had the same assessment for their school functioning scale. On the other hand, parents had lower expectations about their children's quality of life. It was well-noticed that all parents thought that their children had a lower level of quality of life. Thus, parents' reported quality of life was lower than child's reported quality of life.

\section{Statistical Relationships between sample characteristics and levels of depression and anxiety}

We found some statistical relationships between study independent variables and level of quality of life among children in all groups. Some variables are thought to be a cause for low scores in some scales among children. For instance, low scores in the emotional scale were evident in children who are 
hospitalized more frequently. And it was noticed that, as days of hospitalization increase per week, scores become lower. On the other hand, child's nor parents' education seemed to affect child's life. However, good levels of parents' education helped them for better understanding of their children's health status. Another important variable that affected children is duration of diagnosis. It was observed that children with longer duration suffered more than children who were diagnosed newly. In addition, family economic status had an impact on child's mental health. Children of families with low income were more anxious than children of families with high income. Nevertheless, family size has a positive effect on child's status as the child has better support from his or her siblings.

\section{Multivariate regression analysis}

Multivariate analysis revealed that hospitalization, diagnosis duration and family income were independent factors positively associated with quality of life among children in all groups of study sample.

\section{Discussion}

Quality of life assessment for patients with chronic conditions is very essential since they are prone to many changes in their lives after getting diagnosed. Children are a vulnerable group and significantly affected by their chronic conditions. The impact of the change in their lives affects not only the child, but the whole family. Our study included three groups of children with different chronic conditions. Initially, it was thought that children with cancer would have the lowest levels of quality of life, but after the completion of the study, it was found that thalassemic children were the lowest. Authors think that this could be explained as follow. Children with thalassemia had their siblings may also have the same disease, which may be a contributory factor to affect their quality of life. On the other hand, children with cancer are sometimes offered treatment outside Gaza Strip. This, in turn, may help them to live better. Children undergoing hemodialysis spent a lot of time connected to dialysis machine. This must be taken into account since this affects these children lives.

Results showed that parents think that their children had a lower level of quality of life than what child thinks. However, parents were very supportive to their children.

The assessment of quality of life among children included in this study could not be only explained in light of their diseases. Family economic situation was a strong factor to affect child's assessment scores. Furthermore, the general status in Gaza Strip may also contribute to the low levels of quality of life, in general.

A study conducted in the same settings as ours by Salah $M$ and his colleagues ${ }^{[14]}$, but limited to pediatric with cancer only, found that most of the participants had a medium level of quality of life with the mean score was 52.53\% using Pediatric Quality of Life Inventory (PedsQL 4.0 generic core scale). They recommended to raise awareness among health care professionals on the importance of communication skills. Another study conducted in Egypt, also among children with cancer, in which authors used 
PedsQL ${ }^{T M}$ 3.0 Cancer Module ${ }^{[15]}$. They identified long duration of hospital admission, similar to our results, is associated with poorer quality of life. Similar factors were identified to affect thalassemic children in Saudi Arabia ${ }^{[16]}$. These factors were family income and family history of thalassemia, also similar to our study.

Most literature included only one group of children or compared children with chronic condition to a control group. Thalassemic children were included in many regional studies. In Qatar, 40 children aged 14-18 diagnosed with thalassemia major were assessed using PedsQL ${ }^{\text {TM }} 4.0$ generic core scale. Those children had significantly lower levels of quality of life compared to healthy matched children ${ }^{[17]}$. A metaanalysis of 26 studies in which thalassemic children were targeted concluded that thalassemia adversely affects health-related quality of life of these children. Furthermore, quality of life should be considered as an essential part of overall health assessment of these patients ${ }^{[18]}$.

Children with cancer were the main focus of most of studies worldwide. A cross-sectional study involved 150 children aged between $7-18$ years without mental illness ${ }^{[19]}$. The study found that children aged 16-18 reported lower school functioning but more sleep and fatigue. By contrast to our study, increasing years since diagnosis was associated with better quality of life. On the other side, some studies assessed the effect of caregivers' distress on health-related quality of life of children with cancer. It was found that caregivers' distress is an important factor in poor health-related quality of life among children with cancer [20].

Quality of life is an important aspect in any patient with chronic condition. This aspect is not welladdressed among children undergoing hemodialysis. However, it is assessed among some other chronic renal conditions and among children with renal transplant. Splinter et al. assessed health-related quality of life among three groups of children with different modalities of treatment (preemptive transplant, nonpreemptive transplant, and dialysis) ${ }^{[21]}$. Apart from treatment modality, all children had significantly lower mean scores and consequently higher proportions of impaired health-related quality of life on almost all domains compared to the healthy norm and other chronic health conditions. A new determinant was identified to affect quality of life was the presence of another comorbidity. Children with chronic kidney disease (CKD) suffer from poor quality of life as years pass. This was concluded by Dotis $\mathrm{J}$ et al. ${ }^{[22]}$ following the assessment of 55 children with CKD. They used Greek version of the KIDSCREEN52 multidimensional questionnaire in children with renal transplant, CKD and a control group. Children in the case group suffered mostly in the physical scale compared to control group.

Thus, to the best of our knowledge, this is the first study to compare quality of life among three different groups of children with chronic conditions.

\section{Conclusion}

Poor quality of life was identified among children included in this study especially thalassemic children. This study showed also that oncology pediatric are not the most affected group by their condition. The 
findings support that poor quality of life among children with chronic conditions should receive more attention in our local medical settings. Furthermore, we recommend the initiation of qualitative research with these groups as they need more space to talk and express their feelings more and more.

\section{Limitation}

The cross-sectional study design limited our ability to uncover causal relationships between healthrelated quality of life and other factors. Another limitation of this study was that we did not include all other psychosocial stressors and we did not investigate potential biological factors (e.g., hormone levels, etc.) in this study.

Furthermore, during the conduction of the study the following difficulties emerged:

1) Interference of external factors such as noise, fatigue of the patients, interruptions by the personnel as the study took place in hospital environment.

2) Study population, children and their parents, sometimes complained from the length of the questionnaire.

\section{Abbreviations}

PedsQL

Pediatric Quality of Life Inventory 4.0 generic core scale

QoL

Quality of Life

TranQoL

Transfusion-dependent Quality of Life

WHO

World Health Organization

SPSS

Statistical Package for Social Sciences

CKD

Chronic Kidney Disease

\section{Declarations}

\section{Authors' contribution}

Afifi $T$ and Elessi $K$ designed the study and prepared the data collection sheet. Data was collected by Baraka O, Omar M, Ahmad I, Al-Attar H, Qudaih A, Alsultan K, Abdelati M, Astal A, Abu Shammala S and Abu-Raus M. Data was coded then entered to SPSS by Baraka O, Obaid M, Albatsh K and Almoqaid A. Afifi $\mathrm{T}$ and Samaan M cleared and analyzed the data. The initial draft was written by Afifi $\mathrm{T}$ and Baraka $\mathrm{O}$. 
Extensive revision to the initial draft was done by Elessi K. Finally, the final draft was prepared by Afifi T. All authors approved the final version of the manuscript.

\section{Acknowledgment}

The authors wish to thank all study participants and wish them better health. In addition, we thank Faculty of Medicine deanery for their support and help to facilitate the conducting of this study.

\section{Availability of data and materials}

The datasets used and/or analyzed during the current study are available from the corresponding author on reasonable request.

\section{Compliance with ethical standards}

\section{Funding}

None

\section{Competing Interest}

Authors declare that they have no conflict of interest.

This research involves human participants who were interviewed after an informed consent and ethical consideration from Ministry of Health in Gaza, Palestine. Participants were answering questions only, NO intervention was done. Thus, this article does not contain any studies with human participants or animals performed by any of the authors.

\section{Ethical consideration and informed consent}

An approved permission was gained from Ministry of Health in Gaza Strip to conduct interviews with pediatric patients and their parents. Furthermore, informed consents were also gained from parents and any unclear questions will be simplified for the participant.

\section{References}

1. Eiser, C., Eiser, R., Stride, B. Quality of life in children newly diagnosed with cancer and their mothers. Health Qual Life Outcomes. 2005; 3:29.

2. Landolt, M., Vollrath, M., Niggli, F., Gnehm, H., Sennhauser, F. Health-related quality of life in children with newly diagnosed cancer: a one year follow-up study. Health Quality Life Outcomes. 2006; 4:63.

3. Matziou, V., Perdikaris, P., Galanis, P., Dousis, E., Tzoumakas, K. Evaluating depression in a sample of children and adolescents with cancer in Greece. Int Nurs Rev. 2008; 55:314-9.

4. Chaudhry, Z., Siddiqui, S. Health Related Quality of Life Assessment in Pakistani Paediatric Cancer Patients Using PedsQL 4.0 Generic Core Scale and PedsQL Cancer Module. Health and Quality of 


\section{Life Outcomes. 2012; 10:52.}

5. Cadiz, DV., Urzua, MA., Campbell, BM. Quality of Life in Children and Adolescents Surviving Acute Lymphoblastic Leukemia. Revista Chilena De Pediatria. 2011; 82:113-121.

6. Cancer. World Health Organization. Accessed in Jan. 2020. Available from https://www.who.int/health-topics/cancer\#tab=tab_1

7. Annual health report (2017). Ministry of Health, Palestine. Accessed in Jan. 2020. Available from https://www.moh.gov.ps/portal/wp-content/uploads/2018/08/MOH-Annual-Report-2017-Final-9-92018.pdf

8. Christopher, J L Murray. Global, regional, and national age-sex specific all-cause and cause-specific mortality for 240 causes of death, 1990-2013: a systematic analysis for the Global Burden of Disease Study 2013. Lancet. 2015; 385(9963): 117-171.

9. Al Sabbah, H., Khan, S., Hamadna, A., Abu Ghazaleh, L., Dudin, A., Karmi, BA. Factors associated with continuing emergence of $\beta$-thalassemia major despite prenatal testing: a cross-sectional survey. International Journal of Women's Health. 2017; 9 673-679.

10. Yasmeen, H., Hasnain, S.Quality of Life of Pakistani Children with $\beta$-Thalassemia Major.Hemoglobin. 2018;42:5-6, 320-325.

11. Alhusaini, OA., Wayyani, LA., Dafterdar, HE., Gamlo, MM., Alkhayat, ZA., Alghamdi, AS., Safdar, OY. Comparison of quality of life in children undergoing peritoneal dialysis versus hemodialysis. Saudi Med J. 2019 Aug;40(8):840-843.

12. 12. Introducing the WHOQOL instruments. WHOQOL: Measuring Quality of Life. World Health Organization. Accessed in Jan. 2020. Available from https://www.who.int/healthinfo/survey/whoqol-qualityoflife/en/

13. Massad, SG., Nieto, FJ., Palta, M. et al.Health-related quality of life of Palestinian preschoolers in the Gaza Strip: a cross-sectional study. BMC Public Health. 2011;11,253

14. Salah, M., Abu Reyala, M., Al Jerjawy, M. Quality of Life Among Children with Cancer in Gaza Strip, American Journal of Health Research. Vol. 6, No. 5, 2018, 119-125.

15. Fawzy, M., Saleh, M., El-Wakil, M., Monir, Z., Eltahlawy, E. Quality of Life in Egyptian Children with Cancer. Journal of Cancer Therapy. 2013 Aug; Vol. 4 No. 7, 1256-1261.

16. Ayoub, MD., Radi, SA., Azab, AM., Abulaban, AA., Balkhoyor, AH., Bedair, SW., Aljaouni, SK., Kari, JA. Quality of life among children with beta-thalassemia major treated in Western Saudi Arabia. Saudi Med J.2013 Dec;34(12):1281-6.

17. Nashwan, AJ., Yassin, MA., Babu, GDJ., Nair, SLK., Libo-On, IL., Hijazi, HA., De Sanctis, V., Soliman, A. Quality of life among adolescents aged 14 to 18 years with Beta-Thalassemia Major (TM) in Qatar. Acta Biomed. 2018 Feb 16;89(2-S):16-26.

18. Arian, M., Mirmohammadkhani, M., Ghorbani, R., Soleimani, M. Health-related quality of life (HRQoL) in beta-thalassemia major ( $\beta$-TM) patients assessed by 36-item short form health survey (SF-36): a meta-analysis. Qual Life Res.2019 Feb;28(2):321-334. 
19. Pan, HT., Wu, LM., Wen, SH. Quality of Life and Its Predictors Among Children and Adolescents with Cancer. Cancer Nurs.2017 Sep/Oct;40(5):343-351.

20. Pierce, L., Hocking, MC., Schwartz, LA., Alderfer, MA., Kazak, AE., Barakat, LP. Caregiver distress and patient health-related quality of life: psychosocial screening during pediatric cancer treatment. Psychooncology.2017 Oct;26(10):1555-1561.

21. Splinter, A., Tjaden, LA., Haverman, L., Adams, B., Collard, L., Cransberg, K., van Dyck, M., Van Hoeck, KJ., Hoppe, B., Koster-Kamphuis, L., Lilien, MR., Raes, A., Taylan, C., Grootenhuis, MA., Groothoff, JW. Children on dialysis as well as renal transplanted children report severely impaired health-related quality of life. Qual Life Res. 2018 Jun;27(6):1445-1454.

22. Dotis, J., Pavlaki, A., Printza, N., Stabouli, S., Antoniou, S., Gkogka, C., Kontodimopoulos, N., Papachristou, F. Quality of life in children with chronic kidney disease. Pediatr Nephrol.2016 Dec;31(12):2309-2316.

\section{Tables}




\section{Table 1: characteristics of study participants}

\begin{tabular}{|c|c|c|c|c|}
\hline \multicolumn{2}{|l|}{ Study variables } & \multirow{2}{*}{$\begin{array}{l}\text { Children with } \\
\text { cancer } \\
(n=50)\end{array}$} & \multirow{2}{*}{$\begin{array}{l}\text { Children with } \\
\text { thalassemia } \\
(n=30)\end{array}$} & \multirow{2}{*}{$\begin{array}{l}\text { Children undergoing } \\
\text { hemodialysis } \\
(n=30)\end{array}$} \\
\hline & & & & \\
\hline \multirow[t]{2}{*}{ Gender } & Male & 31 & 16 & 18 \\
\hline & female & 19 & 14 & 12 \\
\hline \multirow[t]{2}{*}{ Child education } & Yes & 47 & 30 & 27 \\
\hline & No & 3 & 4 & 3 \\
\hline \multicolumn{2}{|c|}{$\begin{array}{l}\text { Child hospitalization in days per } \\
\text { week }\end{array}$} & $4(2)$ & 2 & $4(3)$ \\
\hline \multicolumn{5}{|c|}{ (Mean (standard deviation)) } \\
\hline \multicolumn{2}{|c|}{ Diagnosis duration } & $3(2)$ & $9(4)$ & $4(3)$ \\
\hline \multicolumn{5}{|c|}{ (Mean (standard deviation)) } \\
\hline \multicolumn{2}{|l|}{ Family size } & $7(2)$ & $5(2)$ & $9(2)$ \\
\hline \multicolumn{5}{|c|}{ (Mean (standard deviation)) } \\
\hline \multicolumn{2}{|l|}{ Parent's age } & $36(7)$ & $38(4)$ & $36(6)$ \\
\hline \multicolumn{5}{|c|}{ (Mean (standard deviation)) } \\
\hline \multirow{3}{*}{$\begin{array}{l}\text { Parent level of } \\
\text { education }\end{array}$} & No school & 5 & 9 & 0 \\
\hline & Secondary & 21 & 14 & 18 \\
\hline & University & 4 & 7 & 12 \\
\hline \multirow{2}{*}{$\begin{array}{l}\text { Parent } \\
\text { employment }\end{array}$} & Yes & 22 & 13 & 21 \\
\hline & No & 28 & 17 & 9 \\
\hline \multirow{3}{*}{$\begin{array}{l}\text { Family } \\
\text { income }\end{array}$} & $\mathrm{w}$ & 32 & 14 & 19 \\
\hline & termediate & 15 & 14 & 4 \\
\hline & gh & 3 & 2 & 7 \\
\hline
\end{tabular}




\begin{tabular}{|lll|}
\hline \multicolumn{3}{|l|}{ Table 2: multivariate analysis of factors affecting depression } \\
\hline Factors & Odds ratio (95\% Confidence Interval) & P value \\
\hline Hospitalization & 4.014 & 0.040 \\
& $(1.066$ to 15.112$)$ & \\
\hline Diagnosis duration & 4.129 & 0.028 \\
& $(1.168$ to 14.601$)$ & \\
\hline Family income & 4.027 & 0.022 \\
\hline & $(1.074$ to 15.003$)$ & \\
\hline
\end{tabular}

\section{Figures}

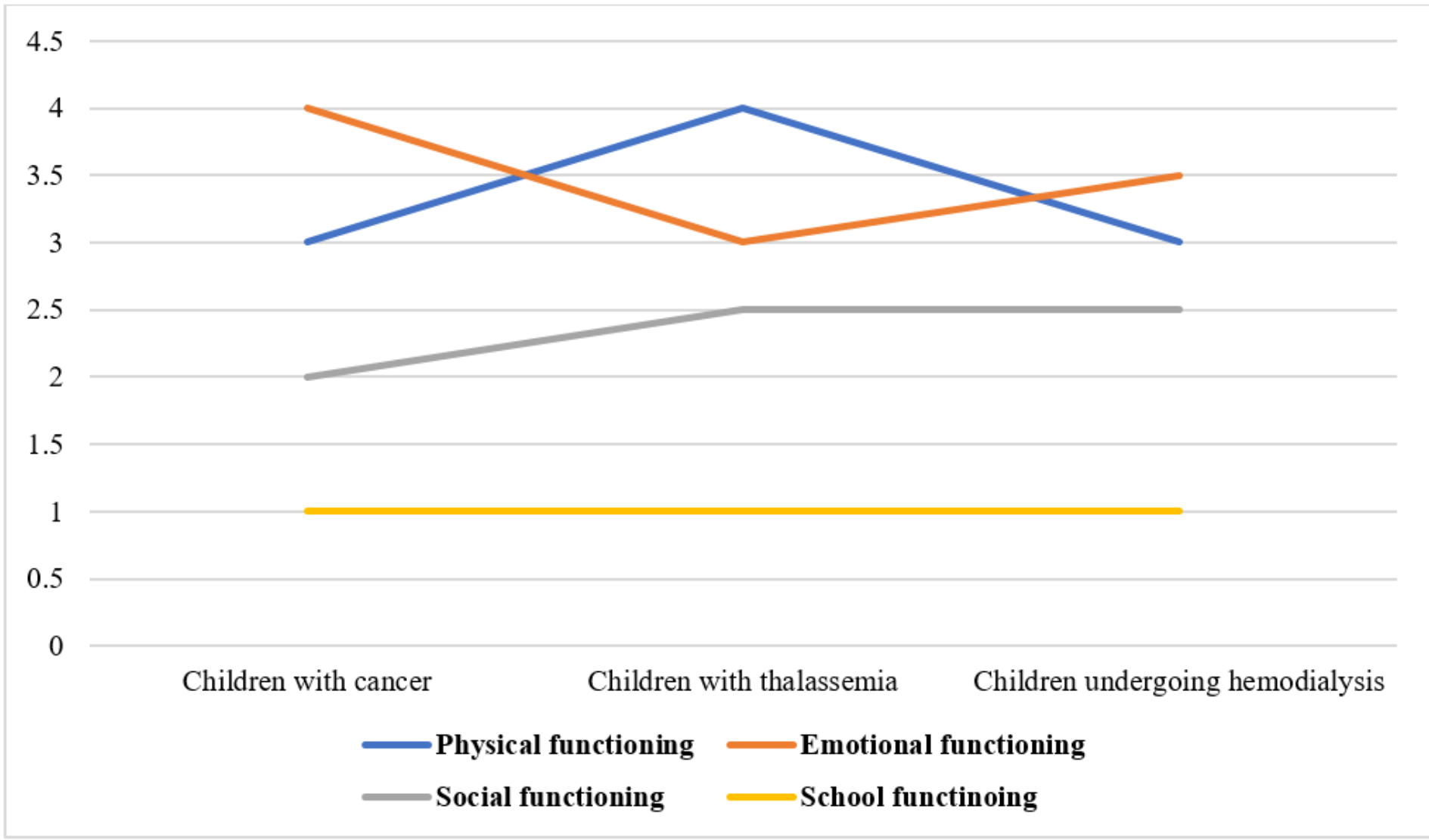

\section{Figure 1}

Mean values of children's scores in PedsQL 4.0 Generic Core Scales 